\title{
An Assortment of Devices for Effecting Intelligent Tutors and Simulated Classrooms
}

\author{
Ade G. Ola, Xue Bai, Emmanuel E. Omojokun, Adeyemi A. Adekoya \\ Department of Management Information Systems \\ Virginia State University \\ Petersburg, USA
}

\begin{abstract}
The focus of this paper is on devices and implements that may be deployed to realize technology-driven instructors and simulated classrooms. It discusses the barriers to building systems with the capability to replicate the human instructor in simulated learning environments. The paper also presents a confluence of technological advancements and open standards that are primed to facilitate the implementation of the capabilities required for the delivery of individualized instruction in simulated classrooms. The goal of the research being presented is to develop tools and applications that can be used to implement learning environments consisting of technology-driven and persistent instructors, learning objects, and simulated classrooms where learners can engage in learning individually while also benefiting from group learning activities.
\end{abstract}

\section{Introduction}

The ability to provide individually-tailored instruction by an ever-present instructor in a simulated classroom has application in many areas, including in the education of diverse learning groups, in providing instruction in subject areas with teacher shortages, in providing individualized learning environments, and in implementing individualized education plans for students with disabilities. The main goal of the research being presented is to employ open and interoperability standards to develop applications and tools that will lower the cost and effort threshold in constructing systems with the capability to replicate, effectively, domainspecific human instructors and to provide individualized instruction as dictated by the learner's knowledge gaps-knowledge gaps that are discovered during the course of instruction and learning. To meet the overall goal of implementing simulated classrooms and intelligent tutors, solutions are required for these interrelated technical challenges and barriers namely:

- Devising methods and software to simplify construction of domain-specific learning environments
- Achieving learning outcomes that are in-line with an effective one-on-one human instructor

- Allowing free-flowing dialog and interaction between the learner and the technologydriven instructor

- Chronicling history of learning activities of students

- Providing mechanisms to assess, store and access competency, learning outcomes, knowledge, skills and abilities of learners

- Determining and assembling the appropriate instruction for the learner in real time

- Providing tools to implement virtual classrooms that permit student-centered active learning strategies and classroom activities that research studies have shown to be effective

\section{Summary of Pertinent Technologies}

There has been a confluence of technological advancements and open standards that lend themselves to the implementation of the capabilities required for delivering individualized instruction and for enabling rich and free-form dialog and interaction between the learner and the technology-driven instructor. The key technologies are summarized in this section.

\subsection{IMS Learning Design}

The IMS Learning Design provides the framework for implementing instructional and learning strategies and techniques-including strategies and techniques that dictate the kinds of learner-instructor interactions and group interactions that are permitted [1]. A learning design lecture may consist of a sequence of learning units in which learners can listen to classical classroom lectures, take quizzes, join a discussion on specific topics or participate in synchronized group activities. Learning Design (LD) provides features-properties and conditions - which can be used to store information and place conditions on learning design. Conditions may be specified on group activities to ensure that an individual member of a group completes sections of a learning resource before group assignment or discussion can begin. To implement LD conditions, 
the runtime environment records completion of the prerequisite activities and synchronizes access to the next set of activities. Learning designs may also include notifications or messaging. Notifications may be used to trigger new activities during the learning process.

\subsection{SCORM Learning Objects and Environments}

The collection of standards and specifications, Sharable Content Object Reference Model (SCORM) provides a rule-based model for defining rules that describe intended sequence and ordering of learning activities [3] [4] [5]. In addition, SCORM allows constructs for every common form of instructional object (lectures, acclaimed instructional videos, assessments, etc.), which can be assembled in various ways to meet the needs of the individual learner. SCORM-based learning objects and environments provide strong support for individualized instruction, learner-instructor interaction, sequencing of instruction, and communication with external systems and resources.

2.2.1. SCORM Content: SCO. In SCORM, an asset is the basic form of learning resource which does not communicate with a Learning Management System (LMS). Ordinarily, when a learner goes through a learning experience or learning resource, the learner's responses and actions are transmitted to an LMS. But there are cases when it is not necessary to record the learner's experiences; in such cases the experience may be a simple Web page, a Flash object or a WAV audio. However, more often than not, learners' experiences need to be monitored and tracked; SCO is the basic unit of resource used in those cases. A SCO is the lowest level of unit of learning resource that is tracked by the LMS. It is a collection of one or more Assets representing a single launch-able learning experience that communicates with a LMS.

2.2.2. SCO as Assessments. A SCORM assessment is really just another SCO or a set of SCOs [12] [14]. SCORM does not distinguish between content and assessment, it is up to the instructional designer to define measureable learning objectives and to design assessments to measure the objectives whether during a test. An assessment SCO may be designed as single $\mathrm{SCO}$ - in which case the $\mathrm{SCO}$ will consist of many test items. An assessment may also be made of multiple SCOs, where each test item is a SCO. An assessment, which is designed to match learning objective(s), typically reports on learner performance. An assessment may also launch remediation content based on the performance of the learner as s/he goes through the SCO. The key to providing appropriate remediation is the capability to determine the skills or knowledge gap

2.2.3. CMI Interactions. SCORM specifications allow the LMS to collect and store data about learners and their progress through a SCO [5]. A SCO can store information in the LMS or ask the LMS to report previously stored values. The data model referred to as cmi.interactions can be used to record performance on individual questions in a SCO assessment.

Table 1. Selected cmi.interactions Descriptions

\begin{tabular}{|c|c|}
\hline cmi interactions & Description \\
\hline $\begin{array}{l}\text { cmi.interactions.n } \\
\text { id }\end{array}$ & $\begin{array}{l}\text { (long_identifier_type ): Unique } \\
\text { label for the interaction }\end{array}$ \\
\hline $\begin{array}{l}\text { cmi.interactions.n } \\
\text {.type }\end{array}$ & $\begin{array}{l}\text { (state (true_false, multiple_choice, } \\
\text { fill_in, long_fill_in, matching, } \\
\text { performance, sequencing, likert, } \\
\text { numeric, other), RW) Which type } \\
\text { of interaction is recorded }\end{array}$ \\
\hline $\begin{array}{l}\text { cmi.interactions.n } \\
\text {.objectives._coun } \\
\mathrm{t}\end{array}$ & $\begin{array}{l}\text { (non-negative integer, RO) } \\
\text { Current number of objectives (i.e., } \\
\text { objective identifiers) being stored } \\
\text { by the LMS for this interaction }\end{array}$ \\
\hline $\begin{array}{l}\text { cmi.interactions.n } \\
\text {.objectives.n.id }\end{array}$ & $\begin{array}{l}\text { (long_identifier_type (SPM: } \\
\text { 4000), RW) Label for objectives } \\
\text { associated with the interaction }\end{array}$ \\
\hline $\begin{array}{l}\text { cmi.interactions.n } \\
\text {.timestamp }\end{array}$ & $\begin{array}{l}\text { (time(second,10,0), RW) Point in } \\
\text { time at which the interaction was } \\
\text { first made available to the learner } \\
\text { for learner interaction and } \\
\text { response }\end{array}$ \\
\hline $\begin{array}{l}\text { cmi.interactions.n } \\
\text {.correct_response } \\
\text { s._count }\end{array}$ & $\begin{array}{l}\text { (non-negative integer, } \mathrm{RO} \text { ) } \\
\text { Current number of correct } \\
\text { responses being stored by the } \\
\text { LMS for this interaction }\end{array}$ \\
\hline $\begin{array}{l}\text { cmi.interactions.n } \\
\text { correct_response } \\
\text { s.n.pattern }\end{array}$ & $\begin{array}{l}\text { (format depends on interaction } \\
\text { type, RW) One correct response } \\
\text { pattern for the interaction }\end{array}$ \\
\hline $\begin{array}{l}\text { cmi.interactions.n } \\
\text {.weighting }\end{array}$ & $\begin{array}{l}\text { (real }(10,7), \mathrm{RW}) \text { Weight given to } \\
\text { the interaction relative to other } \\
\text { interactions }\end{array}$ \\
\hline $\begin{array}{l}\text { cmi.interactions.n } \\
\text {.learner_response }\end{array}$ & $\begin{array}{l}\text { (format depends on interaction } \\
\text { type, } \mathrm{RW} \text { ) Data generated when a } \\
\text { learner responds to an interaction }\end{array}$ \\
\hline $\begin{array}{l}\text { cmi.interactions.n } \\
\text {.latency }\end{array}$ & $\begin{array}{l}\text { (timeinterval (second,10,2), RW) } \\
\text { Time elapsed between the time the } \\
\text { interaction was made available to } \\
\text { the learner for response and the } \\
\text { time of the first response }\end{array}$ \\
\hline
\end{tabular}

Table 1 gives descriptions of a few of the cmi.interactions that make up the CMI data model. A single interaction describes a test item, and for a single test item, a single interaction can store information such as: type of question; the order of the responses as presented to the learner; and the learner's actual response.

The cmi.interactions model provides the mechanism for tracking and collecting quite an amount of data about the learner during the learning 
process-going through a SCO assessment. The interaction data collected at the SCO level would be more useful if there is a straightforward way of synthesizing the data across SCO and other dimensions such as time.

\subsection{IMS Competency Data Framework}

The capability to determine the knowledge and skills of a learner is a prelude to providing individualized instruction to that learner. Apart from using assessments to measure a learner's skills against learning objectives, individuals possess latent knowledge, skills, and abilities that must be considered in providing individualized instruction. Competency and skills are often recorded as grades. In colleges, prerequisites are conditions of enrollment that students are required to meet prior to enrollment in particular courses or programs. However, students who have successfully completed prerequisite courses are not always ready for the corresponding target courses.

The IMS Reusable Definition of Competency or Educational Objective specification provides a means of creating common understandings of competencies that appear as part of a learning or career plan, as learning pre-requisites, or as learning outcomes [13]. The information model presented in the specification can be used to exchange these definitions between learning systems, human resource systems, learning content, competency or skills repositories, and other relevant systems. The IMS specification deals with cataloguing of competencies, but classification poses more challenge because skills and competencies have to be interpreted within established contexts such as educational levels or occupations.

\subsection{Tools Interoperability Framework and O.K.I. Framework}

The IMS Tools Interoperability guidelines (IMS TI) [2] provide a framework that allows tools to be easily integrated into a Course Learning Environment (CLE). IMS TI enables learning management systems to present external tools sideby-side with their native learning tools. With a base SCORM-conformant Learning Management System (LMS) that leverages the key advantages and properties of SCORM content and environment (i.e., Accessibility, Durability, Adaptability, Affordability, Interoperability, and Reusability), IMS TI allows dynamic, flexible, low cost, and open architecture networks that fully support the current and anticipated suite of training technologies.

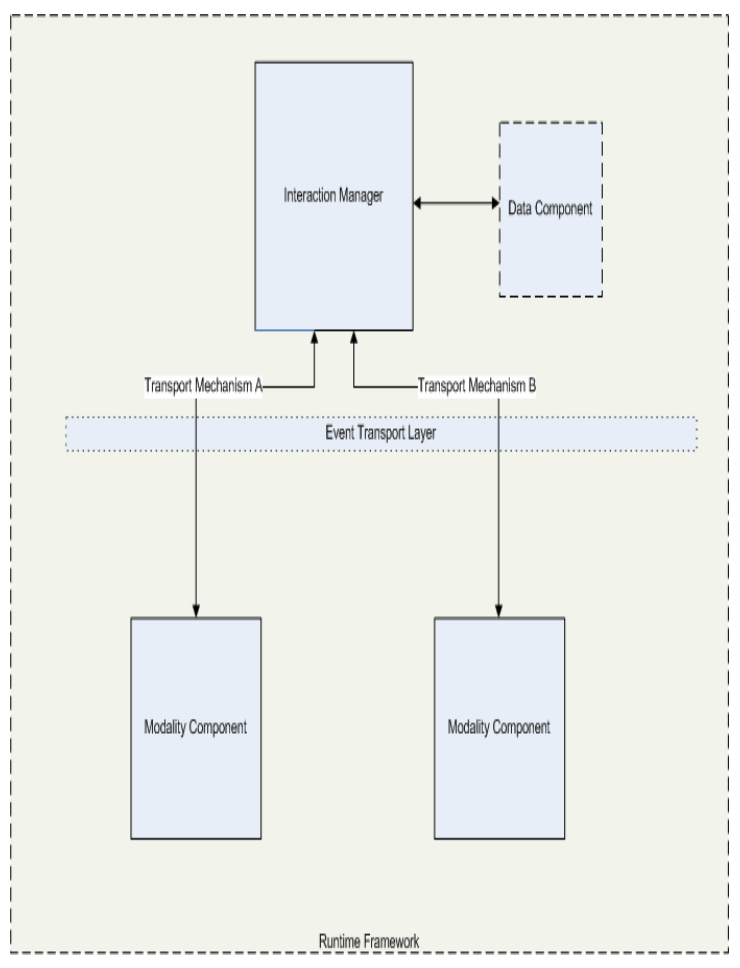

Figure 1. Multimodal Architecture and Interfaces

Software architecture is a framework that defines an organization of system components. The OKI architecture is based on the approach that specifies what services are needed and how software components can reference and provide or use those services-without any reference to the specific technologies used to implement a service. The Campus Project provides a good illustration of the OKI Framework [15]. The approach (of organizing systems around services) is referred to as ServiceOriented Architecture. The OKI architecture is based on service descriptions (referred to as Open Service Interface Definitions (OSIDs)), which are intended to be consistent, well beyond the life-time of the specific technology used to implement the services. Applications that acquire a service do so through the OSIDs, which should not change when a new version of the service is installed. The OSIDs service definitions insulate the applications making use of the services so the service provider can easily be switched. From the perspective of an educational institution, OKI's Service-Oriented Architecture provides an open and extensible framework that enables acquisition of a robust learning environment. The implementation architecture can be depicted as functional boxes fitted together to form a learning system in such a way that there are few dependencies. The architecture allows a system to be easily reconfigured to add or remove tools. 


\subsection{Multimodal Interaction}

The Multimodal Architecture and Interfaces describes the architecture of the Multimodal Interaction (MMI) framework and the interfaces between its constituents [6]. The diagram in figure 1 above taken from depicts the architecture [6]. The constituents of the MMI architecture are presented in the following list:

- The Interaction Manager, which coordinates the different modalities; it is the Controller in the model-view-controller (MVC) paradigm

- The Data Component, which provides the common data model and represents the Model in the MVC paradigm

- The Modality Components, which provide modality-specific interaction capabilities

- The Runtime Framework, which provides the basic infrastructure and enables communication among the other Constituents

The Event Transport Layer is responsible for delivering events among the Interaction Manager and the Modality Components. Because WebSocket provides bi-directional, full-duplex communications channels over a single TCP connection, the technology can be used to implement the Event Transport mechanism. A brief description of WebSocket is presented in the following paragraph.

\subsection{Websocket Protocol}

The WebSocket API is being standardized by the $\mathrm{W} 3 \mathrm{C}$, and the WebSocket protocol has been standardized by the IETF as RFC 6455 [7]. Historically, creating web applications that need bidirectional communication between a client and server (e.g., instant messaging) has required an abuse of HTTP to poll the server for updates while sending upstream notifications as distinct HTTP calls. This results in a variety of problems as listed:

- The server is forced to use a number of different underlying TCP connections for each client: one for sending information to the client and another one for each incoming message

- With each client-to-server message having an HTTP header, the wire protocol has a high overhead

- To track replies, the client-side script is forced to maintain a mapping from the outgoing connections to the incoming connections

A simpler solution would be to use a single TCP connection for traffic in both directions - this is what the Websocket Protocol provides. WebSocket has been shown to provide a 500:1 or-depending on the size of the HTTP headers - even a 1000:1 reduction in unnecessary HTTP header traffic and a 3:1 reduction in latency [8].

\subsection{InkML for Handwriting Interaction}

Up till recently, ink input formats have been proprietary. However, the Ink Markup Language is an open format for representing ink data entered with an electronic pen or stylus [9]. Open InkML ink standard and implementations are key technologies that will permit the learner to show intermediate steps, which can be used to determine the skills and misconceptions of the learner. Using dynamic handwritten notes (i.e., notes where the actual trace of the written characters is shown), rather than static notes, allows synchronization of notes with audio narration and other objects of various media types. Young learners are expected to benefit from dynamic handwritten notes because the presentation will mimic the typical classroom teacher who is writing on the blackboard.

The primitives of InkML permit ink data to be organized in a variety of ways, including as Archival InkML, which allows documents to be stored for later retrieval or processing, and as Streaming InkML, where ink data may be transmitted in real time as applications exchange ink messages. The fundamental data element in an InkML file is the $<$ trace>. A trace represents a sequence of contiguous ink points - e.g., the $\mathrm{X}$ and $\mathrm{Y}$ coordinates of the pen's position. A sequence of traces accumulates to form within a trace. Unlike today's collaborative whiteboards, which typically use complex or closed protocols for communication and are not interoperable across multiple platforms, InkML represents digital ink in a form that allows both transmission and higher-level semantic analysis [10].

\subsection{Knowledge Representation with Ontology}

The application of Ontology (and the associated standard, OWL 2) to domain knowledge representation in education and training should make it possible for human actors to understand how instructional objects relate to domain concepts [11]. It should also permit more precise cognitive models of student performance and skills. The capability to attach varying representation of an instructional object can permit differentiated instruction according to students' learning-style preferences and profile. The use of predefined templates for SCORMconformant instructional objects and the ability to attach objects to domain concepts in ontology maps ensures that anyone with the understanding of the concepts and the nomenclature of the domain will be able to put together materials that will meet specific purposes. 


\subsection{Modeling of Learning History and Mining Learning History Data}

Learning and educational data abound in various formats and platforms. What is needed is a sound logical model upon which useful tools for schools and learning can be implemented. Such a model will facilitate development of tools capable of chronicling the history of students' learning, analyzing the performance history of individual students across multiple dimensions, calculating individual students' mastery of the curriculum, and recommending necessary changes to learning plans for the respective students. A non-profit organization in the United States, inBloom Inc, is leading the effort to develop a data store model that will support interoperability of tools in elementary and secondary school (K12) environments. InBloom provides for developers an API-enabled data store and a set of tools for data interoperable environments in $\mathrm{K} 12$, so as to make personalized learning possible.

These key components of inBloom include the following [16]:

- Data Store: Secure data management service that allows States and school districts to bring together and manage student and school data and connect it to learning tools used in classrooms.

- APIs: Provide authorized applications and school data systems with access to the Data Store.

- Learning Registry Index (LRI): Provides valuable data about learning resources and learning objectives to inBloom-compatible applications. With LRI, there are tools to tag and discover content: Tagger allows publishers and content managers to tag learning resources using criteria such as learning objectives so users can find the content with the Search or the Browser tools.

But perhaps the most significant aspect of inBloom technology is the comprehensive presentation of data items and entities or dimensions across the many domains in the school environment. Data cubes and dimensional data model are candidates for storing and analyzing learning data. The central structure of the dimensional model is the cube-hypercube to be exact. The edges of a cube consist of dimensions, where each dimension represents a real-world entity in the environment being modeled. The cells in the data cube represent the measure of interest.
While the information presented in the dimensional cube model do exist in the source systems (such as a relational database), the Cube representation depicts information in a form that is easily understood because it uses the semantics of the business directly. To use the language in Data Cube Vocabulary, a data cube is organized according to a set of these components: dimensions, attributes and measures [17]. The attribute components allow the observed value(s) to be qualified and interpreted. They enable specification of the units of measure, any scaling factors and metadata such as the status of the observation (e.g. estimated, provisional).

The dimension components are used to identify the observations, and the measure components represent the phenomenon being observed. There are two approaches to representing multiple measures supported by the Data Cube vocabulary. In the first approach, an additional dimension is introduced and each observation records a single observed value for one measure. In the second approach a single observation can provide values for multiple different measures.

\section{Matching Solution Approaches to Technical Barriers}

\subsection{Devising Devices to Simplify Construction of Domain-specific Technology- driven Instructors}

The task here is to devise solutions that would generalize to multiple domains, be based on open architecture, and be able to represent domain knowledge so as to enable instructional content development, permit easy authoring of content, and make it easy to build technology-driven instructors for specific domains. To satisfy these objectives:

- SCORM technologies have been identified as the main platform for content development and content delivery

- Ontology and associated standards will handle domain knowledge representation

- Two tracks of design and development are required: (i) SCORM content editors, curriculum-course tools, ontology editors and tools, performance assessment application, and general tools for multimodal interactions (ii) construction of a technology-driven instructor for specific domains using the applications, tools, and methods developed 


\subsection{Achieving Learning Outcomes that are in line with an Effective One-on-one Human Instructor}

Achieving outstanding learning outcomes depends on a number of factors: capturing full domain knowledge, associating adequate instructional objects to concepts in the domain, allowing quality learner-instructor interaction, conducting assessments, and modeling performanceskills relationships. But here the focus is on implementation of pedagogy and learning strategies and accommodation of learning preferences. The IMS Learning Design framework will be the vehicle for implementing and simulating various classroom learning activities, including the following:

- Learner engagement strategies (such as quote minus one, guided/interactive notes, documented problem solutions, make them guess, frequent assessment), which have been shown to be effective, needs to be implemented

- Classroom activities (attend class, listen to lectures, take notes including using guided notes, pose questions, answer questions, etc.) that have been shown to be effective will be required

- By attaching multiple forms of the same instructional object, instruction will be dictated by the learning style preferences in the learner profile

\subsection{Allowing Free-flowing Dialog and Interaction between the Learner and the Technology-Driven Instructor}

The rule-based model of SCORM which permits sequencing and ordering of learning activities is the key technology for controlling learner-instructor interaction. However, in order to support free-form dialog, it is crucial that the following tools and technologies be employed:

- Content editing tools to facilitate reuse of effective resources from world-class teachers and digital libraries

- $\quad$ Non-proprietary pen input (InkML) to permit lectures that consists of streaming ink synchronized with audio narration, handwritten answers/explanation to questions, and showing of intermediate steps; the ability to show intermediate steps in problem-solving permits more accurate inferences about the skills and misconceptions of the learner

- Handwriting recognition, speech-to-text, and text analysis and data mining are key technologies for free-form dialog between the learner and the intelligent tutor.

- CMI.interaction track hundreds of data items and information as learners progress though a SCORM content, including such information as "time elapsed between the time the interaction was made available to the learner for response and the time of the first response." CMI.interactions could form a basis for free-flowing dialog between the learner and the technology-driven instructor.

\subsection{Determining and Assembling in Real Time the Appropriate Instruction for the Learner}

The task here is to capture domain knowledge fully so that instructional objects, including assessments, can be linked to concepts. To satisfy the objectives:

- Ontology (and the associated standard, OWL 2) will be the vehicle for domain knowledge representation; it is machine readable, yet understandable by the human actors

- Assessments as well as other instructional objects will be attached to concepts; the ability to attach assessments to concepts is vital in determining the instructions that are appropriate

- Inference networks will be used to recommend instruction based on the learner's performance on assessments

- Using historical data of learners' performance, probability distribution functions that depict causal relationships between concepts will be constructed and refined over time

\subsection{Providing Tools to Implement Virtual Classrooms}

The goal here is to implement environments that permit student-centered active learning strategies and classroom activities that research studies have shown to be effective. With IMS Learning Design, one can describe and implement educational activities based on different strategies such as group work and collaborative learning. Learning Design is defined in three levels. Level A defines the basic entities or elements: activities, roles, and learning objects or services.

The specification provides a language for describing how learners and other role players perform activities using learning resources - objects and services - and how the three entities are coordinated into the learning flow. In essence, one or more players in roles (learners, teachers etc.) perform 
a series of activities (assessment, discussion, simulation) in an environment consisting of learning objects or services. Services offer generic functions such as email, conferencing, searching and announcements.

However, to be an effective alternative to the classroom, the usual classroom activities must be accommodated. Thus, the virtual classroom must provide tools that will facilitate the implementation of learning and teaching best practices such as engaging students, performing continuous and frequent assessment of student learning, and incorporating small group activities. The benefit of using the O.K.I. framework as the base infrastructure is that it provides interfaces that support educational services in class administration, content repository, assessment, and communication services. Tools will be needed to accommodate a wide range of instructional requirements and styles. Acquired tools, if they conform to IMS Tools Interoperability (TI) Guidelines, can be integrated easily into O.K.I. collaboration and learning environment. With the IMS TI guidelines, external tools may be attached side-by-side with the native learning tools of a central learning system.

\section{Conclusion}

The goal of this paper is to provide a learning environment where learners would have on going access to learning objects, an ever-present technology-driven instructor, and a simulated classroom setting where the leaner can have the benefits of group learning activities. The ability to provide individually tailored instruction by a personal assistant for learning has application in many areas, including in the education of diverse learning groups, in life-long learning, and in implementing individualized education plans for students with disabilities.

In the paper, candidate devices have been proposed as solutions to identified technical barriers to implementing intelligent tutors and simulated classrooms. The capability to determine the knowledge and skills of a learner poses a great challenge to providing individualized instruction. Apart from using assessments to measure a learner's skills against learning objectives, the greater challenge may be in providing mechanisms to store and access the latent knowledge, skills, and abilities of the learner.

\section{References}

[1] IMS Learning Design Specifications. Available: http://www.imsglobal.org/learningdesign/index.html [April 2, 2013].
[2] IMS Global Learning Consortium. IMS Tools Interoperability Guidelines. http://www.imsglobal.org/ ti/tiv1p0/imsti_guidev1p0.html [April 2, 2013].

[3] SCORM-CAM: SCORM 2004 4th Edition Content Aggregation Model (CAM) Version 1.0. Advanced Distributed Learning (ADL). Available Online: www.adlnet.org [April 2, 2013].

[4] SCORM-SN: SCORM 2004 4th Edition Sequencing and Navigation (SN) Version 1.0 Advanced Distributed Learning (ADL). Available Online: www.adlnet.org [April 2, 2013].

[5] SCORM-RTE: SCORM 2004 4th Edition Run-Time Environment (RTE) Version 1.0 Advanced Distributed Learning (ADL). Available Online: www.adlnet.org [April 2, 2013].

[6] Michael Bodell, Deborah Dahl, Ingmar Kliche, Jim Larson, Brad Porter, Dave Raggett, et al. Multimodal Architecture and Interfaces, W3C Recommendation. Available: http://www.w3.org/TR/mmi-arch/ [April 2, 2013].

[7] I. Fette and A. Melnikov (RFC 6455, 2011). The WebSocket Protocol. Internet Engineering Task Force (IETF) Request for Comments: 6455 http://www.rfceditor.org/rfc/rfc6455.txt [April 2, 2013].

[8] P. Lubbers (2012), HTML5 WebSocket: Full-duplex, Real-time Web Communication. http://refcardz. dzone.com/refcardz/html5-websocket [April 2, 2013].

[9] Watt, S. M. and Underhill (ed.). Ink Markup Language (InkML) W3C Recommendation 20 September 2011. Available: http://www.w3.org/TR/InkML/ [April 2, 2013].

[10] Regmi, A. and Watt, S.M. "A Collaborative Interface for Multimodal Ink and Audio Documents." 10th Int. Conf. on Document Analysis and Recognition, 2009, pp. 901-905.

[11] Boris Motik, Peter F. Patel-Schneider, and Bijan Parsia (Editors). OWL 2 Web Ontology Language Structural Specification and Functional-Style Syntax (Second Edition), W3C Recommendation 11 December 2012: Available online: http://www.w3.org/TR/owlsyntax/ [April 2, 2013].

[12] Advanced Distributed Learning (2011). SCORM Users Guide for Instructional Designers, SCORM 2004 4th Edition, Version 8.http://www.adlnet.gov/ wpcontent/ uploads/2011/12/SCORM_Users_Guide_for_ISDs.pdf [January 19, 2014].

[13] IMS Reusable Definition of Competency or Educational Objective - Information Model Revision: 25 October 2002. http://www.imsglobal.org/competencies/ rdceov1p0/imsrdceo_infov1p0.html [January 19, 2014].

[14] Best Practices for the Design and Development of SCORM Assessments Available online: http://www.jointadlcolab.org/support/design_and_ development/index.aspx [January 19, 2014]. 
International Journal for e-Learning Security (IJeLS), Volume 3, Issue 2, September 2013

[15] The Campus Project, Available online: http://www. campusproject.org/ en/index.php [January 20, 2014].

[16] inBloom (2012) inBloom Data Store Logical Model Available online: https://www.inbloom.org/sites/default/ files/docs-developer/data_model-intro.html[January 2014].

[17] RDF Data Cube Vocabulary (2013). ThRDF Data Cube Vocabulary. W3C Editor's Draft 17 December 2013.

Available online: https://dvcs.w3.org/hg/gld/raw-file/

default/data-cube/index.html\#data-cubes [January 2014]. 